\title{
A Self-Adjusting Spectral Conjugate Gradient Method for Large-Scale Unconstrained Optimization
}

\author{
Yuanying Qiu, ${ }^{1}$ Dandan Cui, ${ }^{2}$ Wei Xue, ${ }^{2}$ and Gaohang $Y u^{2}$ \\ ${ }^{1}$ School of Foreign Languages, Gannan Normal University, Ganzhou 341000, China \\ ${ }^{2}$ School of Mathematics and Computer Sciences, Gannan Normal University, Ganzhou 341000, China \\ Correspondence should be addressed to Gaohang Yu; maghyu@163.com
}

Received 21 February 2013; Accepted 17 March 2013

Academic Editor: Guoyin Li

Copyright (c) 2013 Yuanying Qiu et al. This is an open access article distributed under the Creative Commons Attribution License, which permits unrestricted use, distribution, and reproduction in any medium, provided the original work is properly cited.

This paper presents a hybrid spectral conjugate gradient method for large-scale unconstrained optimization, which possesses a self-adjusting property. Under the standard Wolfe conditions, its global convergence result is established. Preliminary numerical results are reported on a set of large-scale problems in CUTEr to show the convergence and efficiency of the proposed method.

\section{Introduction}

Consider the following unconstrained optimization problem:

$$
\min \left\{f(x) \mid x \in \mathfrak{R}^{n}\right\},
$$

where $f: \Re^{n} \rightarrow \mathfrak{R}$ is a nonlinear smooth function and its gradient is available. Conjugate gradient methods are very efficient for solving (1), especially when the dimension $n$ is large, and have the following iterative form:

$$
x_{k+1}=x_{k}+\alpha_{k} d_{k},
$$

where $\alpha_{k}>0$ is a steplength obtained by a line search, and $d_{k}$ is the search direction defined by

$$
d_{k}= \begin{cases}-g_{k}, & \text { for } k=1, \\ -g_{k}+\beta_{k} d_{k-1}, & \text { for } k \geq 2,\end{cases}
$$

where $\beta_{k}$ is a scalar and $g_{k}$ denotes the gradient of $f$ at point $x_{k}$.
There are at least six formulas for $\beta_{k}$, which are given below:

$$
\begin{array}{ll}
\beta_{k}^{\mathrm{FR}}=\frac{g_{k}^{T} g_{k}}{g_{k-1}^{T} g_{k-1}}, & \beta_{k}^{\mathrm{CD}}=-\frac{g_{k}^{T} g_{k}}{d_{k-1}^{T} y_{k-1}}, \\
\beta_{k}^{\mathrm{DY}}=\frac{g_{k}^{T} g_{k}}{d_{k-1}^{T} y_{k-1}}, & \beta_{k}^{\mathrm{PR}}=\frac{g_{k}^{T} y_{k-1}}{g_{k-1}^{T} g_{k-1}}, \\
\beta_{k}^{\mathrm{HS}}=\frac{g_{k}^{T} y_{k-1}}{d_{k-1}^{T} y_{k-1}}, & \beta_{k}^{\mathrm{LS}}=-\frac{g_{k}^{T} y_{k-1}}{d_{k-1}^{T} g_{k-1}},
\end{array}
$$

where $y_{k-1}=g_{k}-g_{k-1}$ and $\|\cdot\|$ denotes the Euclidean norm. In the above six methods, HS, PR, and LS methods are especially efficient in real computations, but one may not globally converge for general functions. FR, CD, and DY methods are globally convergent, but they perform much worse. To combine the good numerical performance of HS method and the nice global convergence property of DY method, Dai and Yuan [1] proposed an efficient hybrid formula for $\beta_{k}$ which is defined as the following form:

$$
\beta_{k}^{\mathrm{HSDY}}=\max \left\{0, \min \left\{\beta_{k}^{\mathrm{DY}}, \beta_{k}^{\mathrm{HS}}\right\}\right\} .
$$

Their studies suggested that the HSDY method (5) has the same advantage of avoiding the propensity of short steps as the HS method [1]. They also proved that the HSDY method 
with the standard wolfe line search produces a descent search direction at each iteration and converges globally. Descent condition may be crucial for the convergence analysis of conjugate gradient methods with inexact line searches $[2,3]$. Further, there are some modified conjugate gradient methods [4-7] which possess the sufficiently descent property without any line search condition. Recently, Yu [8] proposed a spectral version of HSDY method:

$$
\beta_{k}^{\mathrm{S}-\mathrm{HSDY}}=\max \left\{0, \min \left\{\beta_{k}^{\mathrm{SDY}}, \beta_{k}^{\mathrm{SHS}}\right\}\right\},
$$

where

$$
\beta_{k}^{\mathrm{SDY}}=\frac{\left\|g_{k}\right\|^{2}}{\delta_{k} y_{k-1}^{T} d_{k-1}}, \quad \beta_{k}^{\mathrm{SHS}}=\frac{g_{k}^{T} y_{k-1}}{\delta_{k} y_{k-1}^{T} d_{k-1}},
$$

with $\delta_{k}=y_{k-1}^{T} s_{k-1} /\left\|s_{k-1}\right\|^{2}, s_{k-1}=x_{k}-x_{k-1}$. The numerical experiments show that this simple preconditioning technique benefits to its performance.

In this paper, based on a new conjugate condition [9], we propose a new hybrid spectral conjugate gradient method with $\beta_{k}$ defined by

$$
\beta_{k}^{\mathrm{DS}-\mathrm{HSDY}}=\max \left\{0, \min \left\{\beta_{k}^{\mathrm{DSDY}}, \beta_{k}^{\mathrm{DSHS}}\right\}\right\},
$$

where

$$
\begin{gathered}
\beta_{k}^{\text {DSDY }}=\frac{\left\|g_{k}\right\|^{2}}{\delta_{k} y_{k-1}^{*}{ }^{T} d_{k-1}}, \quad \beta_{k}^{\text {DSHS }}=\frac{g_{k}^{T} y_{k-1}^{*}}{\delta_{k} y_{k-1}^{*}{ }^{T} d_{k-1}}, \\
y_{k-1}^{*}=y_{k-1}+\frac{\max \left\{\vartheta_{k}, 0\right\}}{\left\|s_{k-1}\right\|^{2}} s_{k-1}, \\
\vartheta_{k}=2\left\{f\left(x_{k}\right)-f\left(x_{k-1}\right)\right\}+\left[g\left(x_{k}\right)+g\left(x_{k-1}\right)\right]^{T} s_{k-1} .
\end{gathered}
$$

A full description of DS-HSDY method is formally given as follows.

Algorithm 1 (DS-HSDY conjugate gradient method).

Data. Choose constants $0<\rho<\sigma<1, \mu>1$, and $0 \leq \epsilon \ll 1$. Given an initial point $x_{1} \in R^{n}$, set $d_{1}=-g_{1}$. Let $k:=1$.

Step 1. If $\left\|g_{k}\right\| \leq \varepsilon$, then stop.

Step 2. Determine $\alpha_{k}$ satisfying the standard Wolfe condition:

$$
\begin{gathered}
g\left(x_{k}+\alpha_{k} d_{k}\right)^{T} d_{k}>\sigma g_{k}^{T} d_{k}, \\
f\left(x_{k}+\alpha_{k} d_{k}\right)-f\left(x_{k}\right) \leq \rho \alpha_{k} g_{k}^{T} d_{k} .
\end{gathered}
$$

Then update $x_{k+1}=x_{k}+\alpha_{k} d_{k}$.

Step 3. Compute $g_{k+1}, \delta_{k+1}$ and $\beta_{k+1}^{\text {DS-HSDY }}$. Then update $d_{k+1}$ such as

$$
d_{k+1}=-\frac{1}{\delta_{k+1}} g_{k+1}+\beta_{k+1}^{\text {DS-HSDY }} d_{k}
$$

Set $k:=k+1$ and go to Step 1 .
The rest of the paper is organized as follows. In the next section, we show that the DS-HSDY method possesses a self-adjusting property. In Section 3, we establish its global convergence result under the standard Wolfe line search conditions. Section 4 gives some numerical results on a set of large-scale unconstrained test problems in CUTEr to illustrate the convergence and efficiency of the proposed method. Finally we have a Conclusion section.

\section{Self-Adjusting Property}

In this section, we prove that the DS-HSDY method possesses a self-adjusting property. To begin with, we assume that

$$
g_{k} \neq 0, \quad \forall k \geq 1,
$$

otherwise, a stationary point has been found, and define the two following important quantities:

$$
\begin{gathered}
q_{k}=\frac{\left\|d_{k}\right\|^{2}}{\left(g_{k}^{T} d_{k}\right)^{2}}, \\
\gamma_{k}=-\frac{\delta_{k} g_{k}^{T} d_{k}}{\left\|g_{k}\right\|^{2}} .
\end{gathered}
$$

The quantity $q_{k}$ shows the size of $d_{k}$, where $\gamma_{k}$ is a quantity showing the descent degree of $d_{k}$. In fact, if $\gamma_{k}>0, d_{k}$ is a descent direction. Furthermore, if $\gamma_{k} \geq C$ for some constant $C>0$, then we have the sufficient descent condition

$$
g_{k}^{T} d_{k} \leq-C\left\|g_{k}\right\|^{2}
$$

On the other hand, it follows from (12) that

$$
d_{k}+\frac{1}{\delta_{k}} g_{k}=\beta_{k}^{\text {DS-HSDY }} d_{k-1} \text {. }
$$

Hence

$$
\left\|d_{k}\right\|^{2}=\left(\beta_{k}^{\text {DS-HSDY }}\right)^{2}\left\|d_{k-1}\right\|^{2}-\frac{2}{\delta_{k}} g_{k}^{T} d_{k}-\frac{1}{\delta_{k}^{2}}\left\|g_{k}\right\|^{2} .
$$

Combining $\left|\beta_{k}^{\text {DS-HSDY }}\right| \leq\left|\beta_{k}^{\text {DSDY }}\right| \leq\left|\beta_{k}^{\text {SDY }}\right|$ with (17) yields

$$
\begin{aligned}
\left\|d_{k}\right\|^{2} & =\left(\beta_{k}^{\mathrm{DS}-\mathrm{HSDY}}\right)^{2}\left\|d_{k-1}\right\|^{2}-\frac{2}{\delta_{k}} g_{k}^{T} d_{k}-\frac{1}{\delta_{k}^{2}}\left\|g_{k}\right\|^{2} \\
& \leq\left(\beta_{k}^{\mathrm{SDY}}\right)^{2}\left\|d_{k-1}\right\|^{2}-\frac{2}{\delta_{k}} g_{k}^{T} d_{k}-\frac{1}{\delta_{k}^{2}}\left\|g_{k}\right\|^{2} .
\end{aligned}
$$

Dividing both sides of (18) by $\left(g_{k}^{T} d_{k}\right)^{2}$ and using (7), we obtain

$$
\frac{\left\|d_{k}\right\|^{2}}{\left(g_{k}^{T} d_{k}\right)^{2}} \leq \frac{\left\|d_{k-1}\right\|^{2}}{\left(g_{k-1}^{T} d_{k-1}\right)^{2}}-\frac{2}{\delta_{k}} \frac{1}{g_{k}^{T} d_{k}}-\frac{1}{\delta_{k}^{2}} \frac{\left\|g_{k}\right\|^{2}}{\left(g_{k}^{T} d_{k}\right)^{2}} .
$$

It follows from (19) and the definitions of $q_{k}$ and $\gamma_{k}$ that

$$
q_{k} \leq q_{k-1}+\frac{1}{\left\|g_{k}\right\|^{2}} \frac{2}{\gamma_{k}}-\frac{1}{\left\|g_{k}\right\|^{2}} \frac{1}{\gamma_{k}^{2}} .
$$


Additionally, we assume that there exist positive constants $\gamma$ and $\bar{\gamma}$ such that

$$
0<\gamma \leq\left\|g_{k}\right\| \leq \bar{\gamma}, \quad \forall k \geq 1,
$$

then we have the following result.

Theorem 2. Consider the method (2), (8) and (12), where $d_{k}$ is a descent direction. If (21) holds, there exist positive constants $\xi_{1}, \xi_{2}$, and $\xi_{3}$ such that relations

$$
\begin{gathered}
-g_{k}^{T} d_{k} \geq \frac{\xi_{1}}{\sqrt{k}}, \\
\left\|d_{k}\right\|^{2} \geq \frac{\xi_{2}}{k}, \\
\gamma_{k} \geq \frac{\xi_{3}}{\sqrt{k}}
\end{gathered}
$$

hold for all $k \geq 1$.

Proof. Summing (20) over the iterates and noting that $d_{1}=$ $-g_{1}$, we get

$$
q_{k} \geq \sum_{i=1}^{k} \frac{1}{\left\|g_{i}\right\|^{2}}\left(\frac{2}{\gamma_{i}}-\frac{1}{\gamma_{i}^{2}}\right) .
$$

Since $q_{k} \geq 0$, it follows from (25) that

$$
\frac{1}{\left\|g_{i}\right\|^{2}}\left(\frac{2}{\gamma_{i}}-\frac{1}{\gamma_{i}^{2}}\right) \leq \sum_{i=1}^{k-1} \frac{1}{\left\|g_{i}\right\|^{2}}\left(\frac{2}{\gamma_{i}}-\frac{1}{\gamma_{i}^{2}}\right) .
$$

Equations (21), (26), and $2 / \gamma_{i}-1 / \gamma_{i}^{2} \leq 1$ yield

$$
\frac{1}{\gamma_{k}^{2}}-\frac{2}{\gamma_{k}}-\frac{\bar{\gamma}^{2}}{\gamma^{2}}(k-1) \leq 0
$$

Furthermore, we have

$$
\frac{1}{\gamma_{k}} \leq 1+\sqrt{1+\frac{\bar{\gamma}^{2}}{\gamma^{2}}(k-1)} \leq 1+\frac{\bar{\gamma}^{2}}{\gamma^{2}} \sqrt{k} \leq \frac{2 \bar{\gamma}}{\gamma} \sqrt{k} .
$$

Thus (24) holds with $\xi_{3}=\gamma / 2 \bar{\gamma}$.

Noting that $-g_{k}^{T} d_{k}=\left\|g_{k}\right\|^{2} \gamma_{k}$ and $\left\|d_{k}\right\| \geq\left\|g_{k}\right\| \gamma_{k}$, it is easy to derive that (22) and (23) hold with $\xi_{1}=\xi_{3} \gamma^{2}$ and $\xi_{2}=\xi_{3}^{2} \gamma^{2}$, respectively. Hence the proof is complete.

Theorem 3. Consider the method (2), (8), and (12), where $d_{k}$ is a descent direction. If (21) holds, then for any $p \in(0,1)$, there exist constants $\xi_{4}, \xi_{5}$, and $\xi_{6}>0$ such that, for any $k$, the relations

$$
\begin{gathered}
-g_{i}^{T} d_{i} \geq \xi_{4}, \\
\left\|d_{i}\right\|^{2} \geq \xi_{5}, \\
\gamma_{i} \geq \frac{\xi_{6}}{\sqrt{k}}
\end{gathered}
$$

hold for at least $[p k]$ values of $i \in[1, k]$.

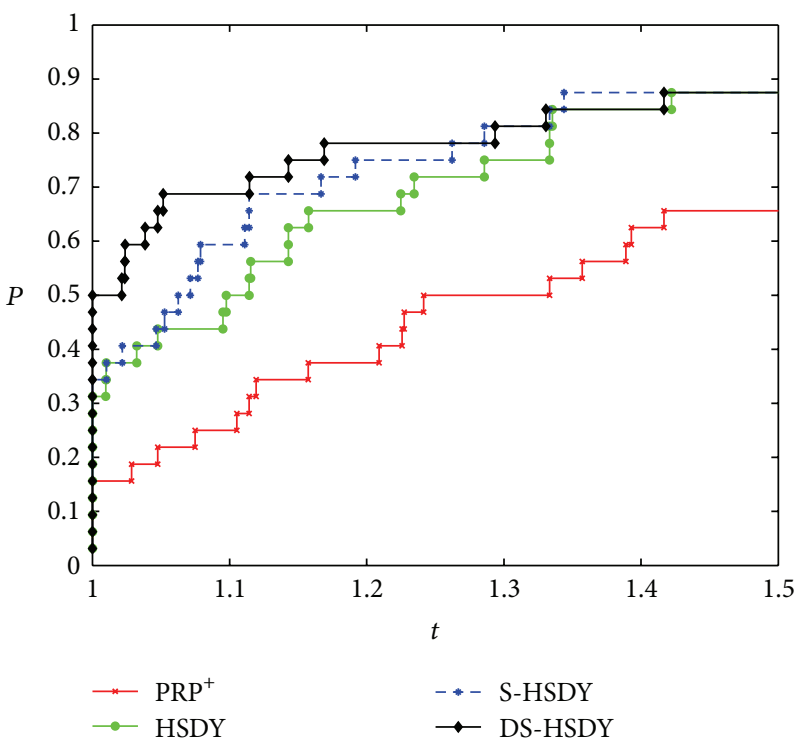

Figure 1: Performance profiles for CPU time.

Proof. The proof is similar to the Theorem 2 in [10], so we omit it here.

Therefore, by Theorems 2 and 3, it was shown that DSHSDY method possesses a self-adjusting property which is independent of the line search and the function convexity.

\section{Global Convergence}

Throughout the paper, we assume that the following assumptions hold.

Assumption 1. (1) $f$ is bounded below in the level set $\mathscr{L}=$ $\left\{x \in R^{n}: f(x) \leq f\left(x_{1}\right)\right\}$;

(2) in a neighborhood $\mathscr{N}$ of $\mathscr{L}, f$ is differentiable and its gradient $g$ is Lipschitz continuous; namely, there exists a constant $L>0$ such that

$$
\|g(x)-g(y)\| \leq L\|x-y\|, \quad \forall x, y \in \mathcal{N} .
$$

Under Assumption 1 on $f$, we could get a useful lemma.

Lemma 4. Suppose that $x_{1}$ is a starting point for which Assumption 1 holds. Consider any method in the form (2), where $d_{k}$ is a descent direction and $\alpha_{k}$ satisfies the weak Wolfe conditions; then one has that

$$
\sum_{k \geq 1} \frac{\left(g_{k}^{T} d_{k}\right)^{2}}{\left\|d_{k}\right\|^{2}}<+\infty
$$

For DS-HSDY method, one has the following global convergence result.

Theorem 5. Suppose that $x_{1}$ is a starting point for which Assumption 1 hold. Consider DS-HSDY method; if $g_{k} \neq 0$ for all $k \geq 1$, then one has that

$$
g_{k}^{T} d_{k}<0 \quad \forall k \geq 1
$$


TABLE 1: Numerical results for $\mathrm{PRP}^{+}$method.

\begin{tabular}{|c|c|c|c|c|c|}
\hline Function & $n$ & $\mathrm{NI}$ & $\mathrm{Nfg}$ & $T(0.01 S)$ & $\|g(x)\|_{\infty}$ \\
\hline Quadratic QF2 & 10000 & 2227 & 2885 & 2016 & $9.98 E-07$ \\
\hline Extended EP1 & 10000 & 4 & 7 & 3 & $6.09 E-13$ \\
\hline Extended Tridiagonal 2 & 10000 & 39 & 98 & 47 & $9.29 E-07$ \\
\hline ARGLINA & 10000 & 5 & 15 & 4 & $1.95 E-07$ \\
\hline ARWHEAD & 10000 & 7 & 14 & 21 & $3.10 E-07$ \\
\hline BDQRTIC & 5000 & 157 & 720 & 526 & $1.47 E-04$ \\
\hline BDEXP & 5000 & 6 & 8 & 7 & $1.72 E-07$ \\
\hline BRYBND & 5000 & 5 & 11 & 1215 & $2.60 E-07$ \\
\hline COSINE & 10000 & 21 & 45 & 39 & $9.60 E-07$ \\
\hline CRAGGLVY & 10000 & 129 & 250 & 444 & $5.35 E-06$ \\
\hline DIXMAANA & 10000 & 6 & 12 & 19 & $4.20 E-07$ \\
\hline DIXMAANB & 10000 & 8 & 16 & 26 & $6.72 E-07$ \\
\hline DIXMAANC & 10000 & 11 & 23 & 38 & $3.38 E-08$ \\
\hline DIXMAAND & 10000 & 13 & 29 & 44 & $1.32 E-07$ \\
\hline DIXMAANE & 5000 & 558 & 799 & 712 & $9.96 E-07$ \\
\hline DIXMAANF & 5000 & 558 & 598 & 525 & $8.65 E-07$ \\
\hline DIXMAANG & 5000 & 519 & 784 & 684 & $5.99 E-07$ \\
\hline DIXMAANH & 5000 & 379 & 3488 & 2469 & $8.97 E-07$ \\
\hline DIXMAANI & 5000 & 593 & 854 & 755 & $7.51 E-07$ \\
\hline DIXMAANJ & 5000 & 492 & 751 & 651 & $5.08 E-07$ \\
\hline DIXMAANK & 5000 & 653 & 979 & 863 & $9.61 E-07$ \\
\hline DQDRTIC & 10000 & 11 & 23 & 19 & $9.59 E-08$ \\
\hline DQRTIC & 10000 & 33 & 57 & 42 & $3.44 E-07$ \\
\hline EDENSCH & 10000 & 26 & 90 & 78 & $9.10 E-06$ \\
\hline EG2 & 10000 & 209 & 1426 & 473 & $1.10 E-03$ \\
\hline ENGVAL1 & 10000 & 30 & 93 & 21 & $1.37 E-06$ \\
\hline EXTROSNB & 10000 & 29 & 63 & 25 & $3.77 E-08$ \\
\hline FREUROTH & 10000 & 61 & 145 & 81 & $2.33 E-07$ \\
\hline LIARWHD & 10000 & 25 & 49 & 36 & $6.54 E-09$ \\
\hline NONDIA & 10000 & 9 & 17 & 17 & $1.03 E-09$ \\
\hline NONDQUAR & 5000 & 1786 & 3258 & 1752 & $7.96 E-07$ \\
\hline NONSCOMP & 10000 & 5001 & 6799 & 9751 & $3.47 E-06$ \\
\hline
\end{tabular}

Further, the method converges in the sense that

$$
\liminf _{k \rightarrow \infty}\left\|g_{k}\right\|=0
$$

Proof. Since $d_{1}=-g_{1}$, it is obvious that $g_{1}^{T} d_{1}<0$. Assume that $g_{k-1}^{T} d_{k-1}<0$. By (10) and the definition of the $y_{k}^{*}$, we have $d_{k-1}^{T} y_{k-1}^{*} \geq d_{k-1}^{T} y_{k-1}>0$, then $\beta_{k}^{\text {DSDY }}>0$. In addition, from (8), we have

$$
0 \leq \beta_{k}^{\mathrm{DS}-\mathrm{HSDY}} \leq \beta_{k}^{\mathrm{DSDY}} \leq \beta_{k}^{\mathrm{SDY}}
$$

Let $\lambda_{k}=\beta_{k}^{\mathrm{DS}-\mathrm{HSDY}} / \beta_{k}^{\mathrm{SDY}}$, then we have $0 \leq \lambda_{k} \leq 1$. By (12) with $k+1$ replaced by $k$, and multiplying it by $g_{k}$, we have

$$
g_{k}^{T} d_{k}=\frac{g_{k-1}^{T} d_{k-1}+\left(\lambda_{k}-1\right) g_{k}^{T} d_{k-1}}{\delta_{k} d_{k-1}^{T} y_{k-1}}\left\|g_{k}\right\|^{2} .
$$

From this and the formula for $\beta_{k}^{\mathrm{SDY}}$, we get

$$
\begin{aligned}
\beta_{k}^{\mathrm{DS}-\mathrm{HSDY}} & =\lambda_{k} \beta_{k}^{\mathrm{SDY}}=\frac{\lambda_{k} g_{k}^{T} d_{k}}{g_{k-1}^{T} d_{k-1}+\left(\lambda_{k}-1\right) g_{k}^{T} d_{k-1}} \\
& =\xi_{k} \frac{g_{k}^{T} d_{k}}{g_{k-1}^{T} d_{k-1}},
\end{aligned}
$$

where

$$
\begin{gathered}
\xi_{k}=\frac{\lambda_{k}}{1+\left(\lambda_{k}-1\right) l_{k-1}}, \\
l_{k-1}=\frac{g_{k}^{T} d_{k-1}}{g_{k-1}^{T} d_{k-1}} .
\end{gathered}
$$

At the same time, if we define

$$
\zeta_{k}=\frac{1+\left(\lambda_{k}-1\right) l_{k-1}}{l_{k-1}-1},
$$


TABLE 2: Numerical results for HSDY method.

\begin{tabular}{|c|c|c|c|c|c|}
\hline Function & $n$ & NI & $\mathrm{Nfg}$ & $T(0.01 \mathrm{~S})$ & $\|g(x)\|_{\infty}$ \\
\hline Quadratic QF2 & 10000 & 1593 & 1902 & 1876 & $7.11 E-07$ \\
\hline Extended EP1 & 10000 & 4 & 7 & 4 & $6.09 E-13$ \\
\hline Extended Tridiagonal 2 & 10000 & 34 & 55 & 32 & $9.40 E-07$ \\
\hline ARGLINA & 10000 & 5 & 15 & 4 & $1.95 E-07$ \\
\hline ARWHEAD & 10000 & 13 & 58 & 71 & $4.42 E-07$ \\
\hline BDQRTIC & 5000 & 171 & 567 & 422 & $6.31 E-04$ \\
\hline BDEXP & 5000 & 6 & 8 & 6 & $1.72 E-07$ \\
\hline BRYBND & 5000 & 5 & 11 & 1222 & $2.60 E-07$ \\
\hline COSINE & 10000 & 21 & 46 & 41 & $8.02 E-07$ \\
\hline CRAGGLVY & 10000 & 109 & 255 & 434 & $1.45 E-06$ \\
\hline DIXMAANA & 10000 & 5 & 10 & 16 & $5.13 E-07$ \\
\hline DIXMAANB & 10000 & 9 & 18 & 29 & $2.21 E-07$ \\
\hline DIXMAANC & 10000 & 10 & 21 & 33 & $5.42 E-07$ \\
\hline DIXMAAND & 10000 & 13 & 29 & 45 & $1.14 E-07$ \\
\hline DIXMAANE & 5000 & 446 & 541 & 493 & $9.24 E-07$ \\
\hline DIXMAANF & 5000 & 389 & 876 & 690 & $9.60 E-07$ \\
\hline DIXMAANG & 5000 & 552 & 660 & 602 & $9.85 E-07$ \\
\hline DIXMAANH & 5000 & 202 & 5106 & 3417 & $4.05 E-04$ \\
\hline DIXMAANI & 5000 & 365 & 450 & 409 & $9.95 E-07$ \\
\hline DIXMAANJ & 5000 & 444 & 532 & 484 & $4.95 E-07$ \\
\hline DIXMAANK & 5000 & 367 & 452 & 410 & $9.77 E-07$ \\
\hline DQDRTIC & 10000 & 8 & 17 & 14 & $6.35 E-07$ \\
\hline DQRTIC & 10000 & 37 & 68 & 48 & $3.40 E-07$ \\
\hline EDENSCH & 10000 & 30 & 99 & 85 & $7.97 E-07$ \\
\hline EG2 & 10000 & 305 & 2811 & 879 & $2.08 E-03$ \\
\hline ENGVAL1 & 10000 & 30 & 52 & 21 & $8.62 E-07$ \\
\hline EXTROSNB & 10000 & 27 & 54 & 21 & $6.20 E-09$ \\
\hline FREUROTH & 10000 & 143 & 283 & 177 & $8.01 E-07$ \\
\hline LIARWHD & 10000 & 32 & 62 & 44 & $1.75 E-07$ \\
\hline NONDIA & 10000 & 7 & 14 & 14 & $4.57 E-07$ \\
\hline NONDQUAR & 5000 & 2049 & 3730 & 2011 & $6.68 E-07$ \\
\hline NONSCOMP & 10000 & 58 & 100 & 98 & $5.03 E-07$ \\
\hline
\end{tabular}

it follows from (39) that

$$
g_{k}^{T} d_{k}=\frac{\zeta_{k}}{\delta_{k}}\left\|g_{k}\right\|^{2}
$$

Then we have by (10), with $k$ replaced by $k-1$, that

$$
l_{k-1} \leq \sigma
$$

Furthermore, we have

$$
1+\left(\lambda_{k}-1\right) l_{k-1} \geq 1+\left(-\frac{1-\sigma}{1+\sigma}-1\right) \sigma=\frac{1-\sigma}{1+\sigma} .
$$

The above relation, (40), (41), and the fact that $\sigma<1$ imply that $g_{k}^{T} d_{k}<0$. Thus by induction, (32) holds.

We now prove (33) by contradiction and assume that there exists some constant $\gamma>0$ such that

$$
\left\|g_{k}\right\| \geq \gamma \quad \forall k \geq 1
$$

Since $d_{k}+\left(1 / \delta_{k}\right) g_{k}=\beta_{k}^{\text {DS-HSDY }} d_{k-1}$, we have that

$$
\left\|d_{k}\right\|^{2}=\left(\beta_{k}^{\text {DS-HSDY }}\right)^{2}\left\|d_{k-1}\right\|^{2}-\frac{2}{\delta_{k}} g_{k}^{T} d_{k}-\frac{1}{\delta_{k}^{2}}\left\|g_{k}\right\|^{2} .
$$

Dividing both sides of (44) by $\left(g_{k}^{T} d_{k}\right)^{2}$ and using (36) and (40), we obtain

$$
\begin{aligned}
\frac{\left\|d_{k}\right\|^{2}}{\left(g_{k}^{T} d_{k}\right)^{2}} & =\xi_{k}^{2} \frac{\left\|d_{k-1}\right\|^{2}}{\left(g_{k-1} d_{k-1}\right)^{2}}-\frac{1}{\left\|g_{k}\right\|^{2}}\left(\frac{2}{\zeta_{k}}+\frac{1}{\zeta_{k}^{2}}\right) \\
& =\xi_{k}^{2} \frac{\left\|d_{k-1}\right\|^{2}}{\left(g_{k-1} d_{k-1}\right)^{2}}+\frac{1}{\left\|g_{k}\right\|^{2}}\left[1-\left(1+\frac{1}{\zeta_{k}}\right)^{2}\right]
\end{aligned}
$$

In addition, since $l_{k-1}<1$ and $\lambda_{k} \leq 1$, we have that $\left(1-\lambda_{k}\right)(1-$ $\left.l_{k-1}\right) \geq 0$, or equivalently

$$
1+\left(\lambda_{k}-1\right) l_{k-1} \geq \lambda_{k}
$$


TABLE 3: Numerical results for S-HSDY method.

\begin{tabular}{|c|c|c|c|c|c|}
\hline Function & $n$ & NI & Nfg & $T(0.01 \mathrm{~S})$ & $\|g(x)\|_{\infty}$ \\
\hline Quadratic QF2 & 10000 & 1582 & 1941 & 1836 & $6.58 E-07$ \\
\hline Extended EP1 & 10000 & 4 & 7 & 3 & $6.09 E-13$ \\
\hline Extended Tridiagonal 2 & 10000 & 34 & 55 & 34 & $9.40 E-07$ \\
\hline ARGLINA & 10000 & 5 & 15 & 3 & $1.95 E-07$ \\
\hline ARWHEAD & 10000 & 13 & 58 & 75 & $5.60 E-07$ \\
\hline BDQRTIC & 5000 & 111 & 526 & 377 & $3.39 E-04$ \\
\hline BDEXP & 5000 & 6 & 8 & 5 & $1.72 E-07$ \\
\hline BRYBND & 5000 & 5 & 11 & 1179 & $2.60 E-07$ \\
\hline COSINE & 10000 & 21 & 46 & 39 & $9.72 E-07$ \\
\hline CRAGGLVY & 10000 & 103 & 189 & 332 & $1.94 E-06$ \\
\hline DIXMAANA & 10000 & 5 & 10 & 15 & $5.13 E-07$ \\
\hline DIXMAANB & 10000 & 9 & 18 & 30 & $2.21 E-07$ \\
\hline DIXMAANC & 10000 & 10 & 21 & 33 & $5.42 E-07$ \\
\hline DIXMAAND & 10000 & 13 & 29 & 43 & $1.14 E-07$ \\
\hline DIXMAANE & 5000 & 422 & 514 & 468 & $9.73 E-07$ \\
\hline DIXMAANF & 5000 & 310 & 792 & 618 & $6.77 E-07$ \\
\hline DIXMAANG & 5000 & 410 & 495 & 449 & $9.83 E-07$ \\
\hline DIXMAANH & 5000 & 217 & 6957 & 4642 & $4.13 E-04$ \\
\hline DIXMAANI & 5000 & 380 & 450 & 417 & $9.96 E-07$ \\
\hline DIXMAANJ & 5000 & 359 & 438 & 402 & $9.95 E-07$ \\
\hline DIXMAANK & 5000 & 404 & 485 & 448 & $6.67 E-07$ \\
\hline DQDRTIC & 10000 & 8 & 17 & 14 & $6.35 E-07$ \\
\hline DQRTIC & 10000 & 37 & 68 & 49 & $3.41 E-07$ \\
\hline EDENSCH & 10000 & 30 & 99 & 84 & $1.54 E-06$ \\
\hline EG2 & 10000 & 242 & 1731 & 570 & $4.25 E-04$ \\
\hline ENGVAL1 & 10000 & 29 & 124 & 22 & $1.78 E-06$ \\
\hline EXTROSNB & 10000 & 27 & 54 & 22 & $3.98 E-09$ \\
\hline FREUROTH & 10000 & 214 & 408 & 260 & $8.08 E-07$ \\
\hline LIARWHD & 10000 & 27 & 54 & 37 & $4.45 E-12$ \\
\hline NONDIA & 10000 & 7 & 14 & 14 & $4.58 E-07$ \\
\hline NONDQUAR & 5000 & 1782 & 3210 & 1738 & $8.99 E-07$ \\
\hline NONSCOMP & 10000 & 58 & 100 & 100 & $5.03 E-07$ \\
\hline
\end{tabular}

which with (37) yields

$$
\left|\xi_{k}\right| \leq 1
$$

By (45) and (47), we obtain

$$
\frac{\left\|d_{k}\right\|^{2}}{\left(g_{k}^{T} d_{k}\right)^{2}} \leq \frac{\left\|d_{k-1}\right\|^{2}}{\left(g_{k-1} d_{k-1}\right)^{2}}+\frac{1}{\left\|g_{k}\right\|^{2}} .
$$

Using (48) recursively and noting that $\left\|d_{1}\right\|^{2}=-g_{1}^{T} d_{1}=$ $\left\|g_{1}\right\|^{2}$,

$$
\frac{\left\|d_{k}\right\|^{2}}{\left(g_{k}^{T} d_{k}\right)^{2}} \leq \sum_{i=1}^{k} \frac{1}{\left\|g_{k}\right\|^{2}} .
$$

Then we get from this and (43) that

$$
\frac{\left(g_{k}^{T} d_{k}\right)^{2}}{\left\|d_{k}\right\|^{2}} \geq \frac{\lambda^{2}}{k}
$$

which indicates

$$
\sum_{k \geq 1} \frac{\left(g_{k}^{T} d_{k}\right)^{2}}{\left\|d_{k}\right\|^{2}}=+\infty
$$

This contradicts the Zoutendijk condition (31). Hence we complete the proof.

\section{Numerical Result}

In this section, we compare the performance of DS-HSDY method to $\mathrm{PRP}^{+}$method [11], HSDY method [1], and SHSDY method [8]. The test problems are taken from CUTEr (http://hsl.rl.ac.uk/cuter-www/problems.html) with the standard initial points. All codes are written in double precision Fortran and complied with $\mathrm{f} 77$ (default compiler settings) on a PC (AMD Athlon XP $2500+$ CPU 1.84 GHz). Our line search subroutine computes $\alpha_{k}$ such that the Wolfe conditions (10) and (11) hold with $\rho=10^{-4}$ and $\sigma=0.5$. We 
TABLE 4: Numerical results for DS-HSDY method.

\begin{tabular}{|c|c|c|c|c|c|}
\hline Function & $n$ & NI & Nfg & $T(0.01 S)$ & $\|g(x)\|_{\infty}$ \\
\hline Quadratic QF2 & 10000 & 1623 & 1978 & 1783 & $9.81 E-07$ \\
\hline Extended EP1 & 10000 & 4 & 7 & 3 & $6.09 E-13$ \\
\hline Extended Tridiagonal 2 & 10000 & 34 & 55 & 30 & $1.95 E-07$ \\
\hline ARGLINA & 10000 & 5 & 15 & 3 & $5.60 E-07$ \\
\hline ARWHEAD & 10000 & 13 & 58 & 70 & $5.60 E-07$ \\
\hline BDQRTIC & 5000 & 165 & 448 & 324 & $2.93 E-03$ \\
\hline BDEXP & 5000 & 6 & 8 & 4 & $1.72 E-07$ \\
\hline BRYBND & 5000 & 5 & 11 & 990 & $2.60 E-07$ \\
\hline COSINE & 10000 & 14 & 38 & 28 & $5.78 E-07$ \\
\hline CRAGGLVY & 10000 & 110 & 150 & 266 & $8.92 E-07$ \\
\hline DIXMAANA & 10000 & 5 & 10 & 16 & $5.17 E-07$ \\
\hline DIXMAANB & 10000 & 9 & 18 & 27 & $2.21 E-07$ \\
\hline DIXMAANC & 10000 & 10 & 21 & 31 & $5.42 E-07$ \\
\hline DIXMAAND & 10000 & 13 & 29 & 44 & $1.10 E-07$ \\
\hline DIXMAANE & 5000 & 410 & 493 & 430 & $9.57 E-07$ \\
\hline DIXMAANF & 5000 & 432 & 546 & 469 & $3.65 E-07$ \\
\hline DIXMAANG & 5000 & 476 & 582 & 505 & $5.89 E-07$ \\
\hline DIXMAANH & 5000 & 442 & 1204 & 7792 & $4.05 E-04$ \\
\hline DIXMAANI & 5000 & 397 & 467 & 408 & $9.45 E-07$ \\
\hline DIXMAANJ & 5000 & 445 & 594 & 503 & $9.66 E-07$ \\
\hline DIXMAANK & 5000 & 403 & 507 & 438 & $9.05 E-07$ \\
\hline DQDRTIC & 10000 & 10 & 21 & 17 & $1.19 E-07$ \\
\hline DQRTIC & 10000 & 35 & 62 & 43 & $9.73 E-07$ \\
\hline EDENSCH & 10000 & 29 & 87 & 70 & $5.74 E-06$ \\
\hline EG2 & 10000 & 251 & 1121 & 381 & $4.02 E-03$ \\
\hline ENGVAL1 & 10000 & 29 & 50 & 19 & $4.26 E-07$ \\
\hline EXTROSNB & 10000 & 65 & 122 & 44 & $7.11 E-07$ \\
\hline FREUROTH & 10000 & 50 & 133 & 67 & $1.59 E-07$ \\
\hline LIARWHD & 10000 & 47 & 94 & 61 & $1.16 E-08$ \\
\hline NONDIA & 10000 & 7 & 14 & 12 & $4.60 E-07$ \\
\hline NONDQUAR & 5000 & 1831 & 3262 & 1665 & $9.66 E-07$ \\
\hline NONSCOMP & 10000 & 73 & 126 & 119 & $5.10 E-07$ \\
\hline
\end{tabular}

use the condition $\left\|g\left(x_{k}\right)\right\|_{\infty} \leq 10^{-6}$ or $\alpha_{k} g_{k}^{T} d_{k}<10^{-20}\left|f\left(x_{k}\right)\right|$ as the stopping criterion. The numerical results are presented in Tables 1, 2, 3, and 4 with the form NI/Nfg/T, where we report the dimension of the problem $(n)$, the number of iteration (NI), the number of function evaluations (Nfg), and the CPU time $(T)$ in 0.01 seconds.

Figure 1 shows the performance of these test methods relative to the CPU time, which were evaluated using the profiles of Dolan and Moré [12]. That is, for each method, we plot the fraction $P$ of problems for which the method is within a factor $t$ of the best time. The top curve is the method that solved the most problems in a time that was within a factor $t$ of the best time. Clearly, the left side of the figure gives the percentage of the test problems for which a method is the fastest. As we can see from Figure 1, DS-HSDY method has the best performance which performs better than S-HSDY method, HSDY method, and the well-known $\mathrm{PRP}^{+}$ method.

\section{Conclusion}

In this paper, we proposed an efficient hybrid spectral conjugate gradient method with self-adjusting property. Under some suitable assumptions, we established the global convergence result for the DS-HSDY method. Numerical results indicated that the proposed method is efficient for large-scale unconstrained optimization problems.

\section{Acknowledgments}

This work was partly supported by the National Natural Science Foundation of China (no. 61262026), the JGZX program of Jiangxi Province (20112BCB23027), and the science and technology program of Jiangxi Education Committee (LDJH12088). The authors would also like to thank the editor and an anonymous referees for their comments and 
suggestions on the first version of the paper, which led to significant improvements of the presentation.

\section{References}

[1] Y. H. Dai and Y. Yuan, "An efficient hybrid conjugate gradient method for unconstrained optimization," Annals of Operations Research, vol. 103, pp. 33-47, 2001.

[2] Y. H. Dai and Y. Yuan, "A nonlinear conjugate gradient method with a strong global convergence property," SIAM Journal on Optimization, vol. 10, no. 1, pp. 177-182, 1999.

[3] Z. X. Wei, G. Y. Li, and L. Q. Qi, "Global convergence of the Polak-Ribière-Polyak conjugate gradient method with an Armijo-type inexact line search for nonconvex unconstrained optimization problems," Mathematics of Computation, vol. 77, no. 264, pp. 2173-2193, 2008.

[4] W. W. Hager and H. Zhang, "A new conjugate gradient method with guaranteed descent and an efficient line search," SIAM Journal on Optimization, vol. 16, no. 1, pp. 170-192, 2005.

[5] L. Zhang, W. Zhou, and D.-H. Li, "A descent modified PolakRibière-Polyak conjugate gradient method and its global convergence," IMA Journal of Numerical Analysis, vol. 26, no. 4, pp. 629-640, 2006.

[6] G. Yuan, "Modified nonlinear conjugate gradient methods with sufficient descent property for large-scale optimization problems," Optimization Letters, vol. 3, no. 1, pp. 11-21, 2009.

[7] G. Yu, L. Guan, and W. Chen, "Spectral conjugate gradient methods with sufficient descent property for large-scale unconstrained optimization," Optimization Methods and Software, vol. 23, no. 2, pp. 275-293, 2008.

[8] G. Yu, Nonlinear self-scaling conjugate gradient methods for large-scale optimization problems [Ph.D. thesis], Sun Yat-Sen University, Guangzhou, China, 2007.

[9] G. Li, C. Tang, and Z. Wei, "New conjugacy condition and related new conjugate gradient methods for unconstrained optimization," Journal of Computational and Applied Mathematics, vol. 202, no. 2, pp. 523-539, 2007.

[10] Y.-H. Dai, "New properties of a nonlinear conjugate gradient method," Numerische Mathematik, vol. 89, no. 1, pp. 83-98, 2001.

[11] J. C. Gilbert and J. Nocedal, "Global convergence properties of conjugate gradient methods for optimization," SIAM Journal on Optimization, vol. 2, no. 1, pp. 21-42, 1992.

[12] E. D. Dolan and J. J. Moré, "Benchmarking optimization software with performance profiles," Mathematical Programming, vol. 91, no. 2, pp. 201-213, 2002. 


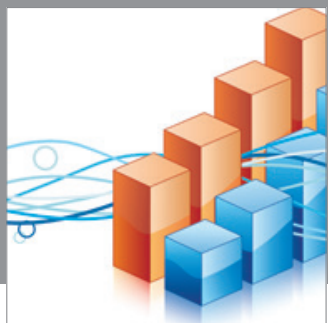

Advances in

Operations Research

mansans

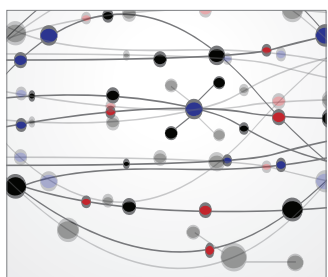

The Scientific World Journal
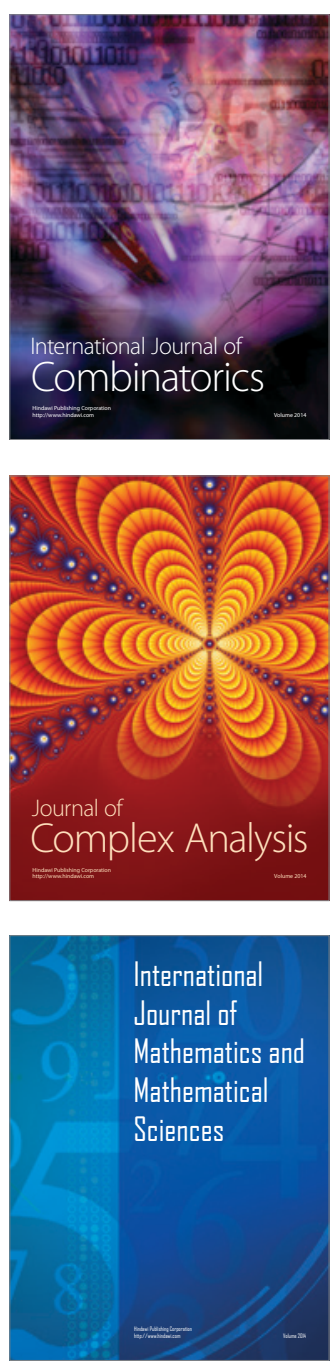
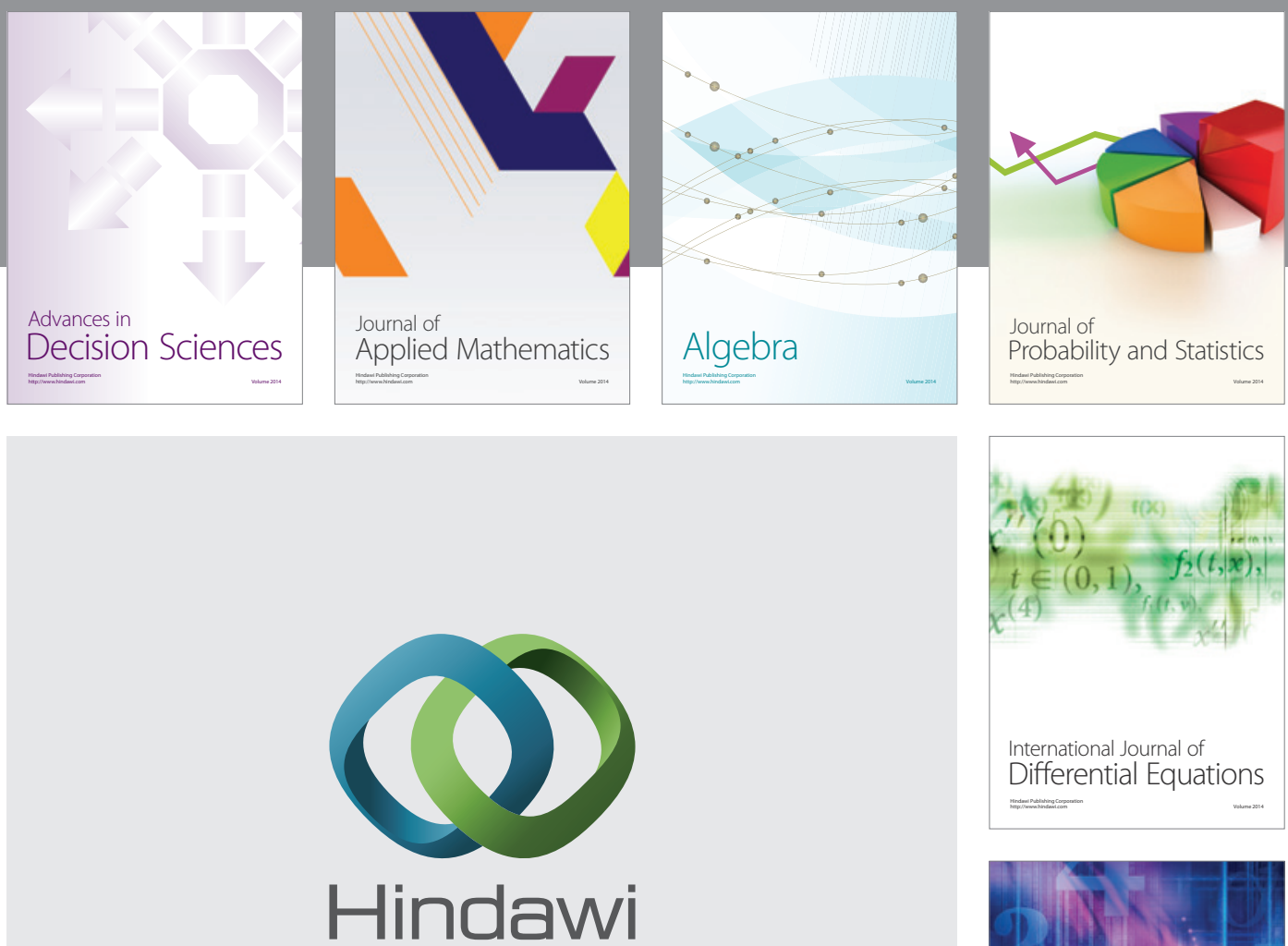

Submit your manuscripts at http://www.hindawi.com
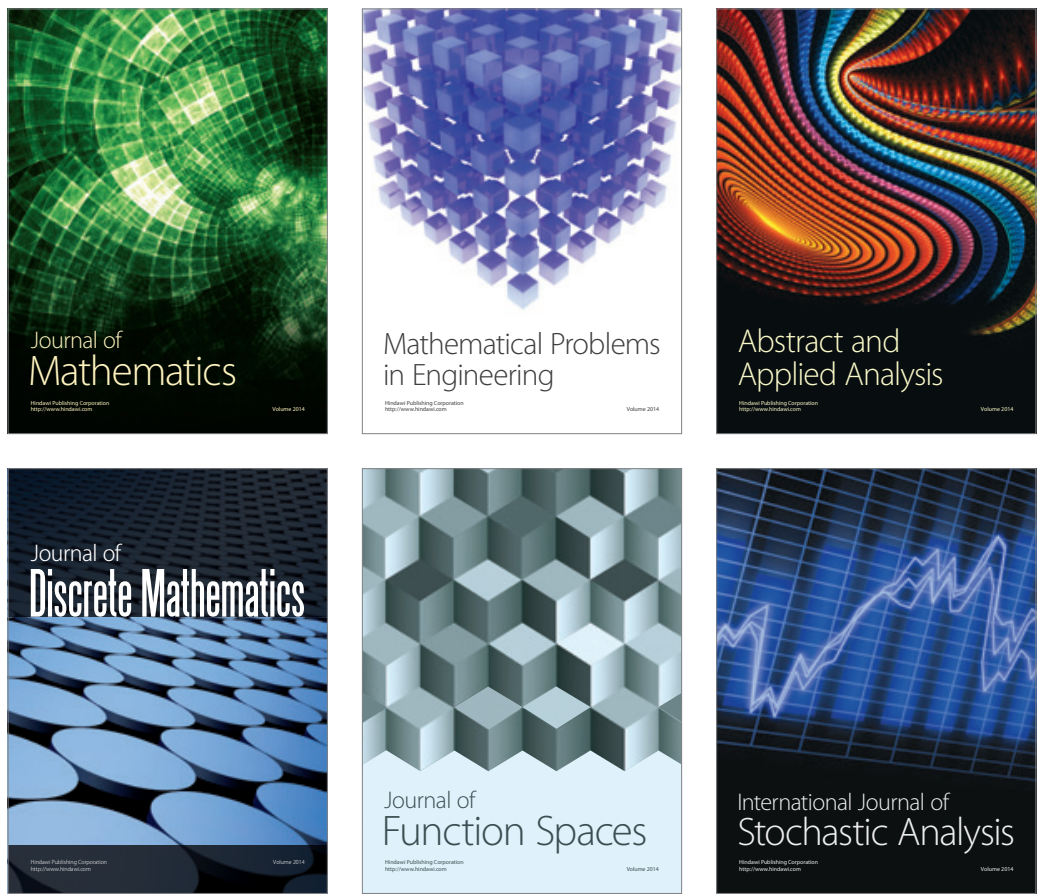

Journal of

Function Spaces

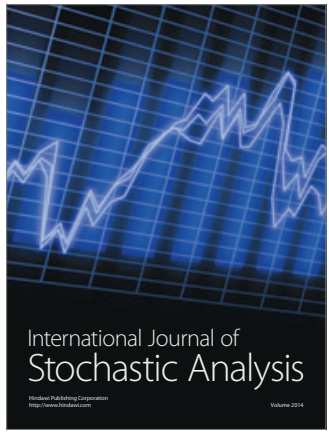

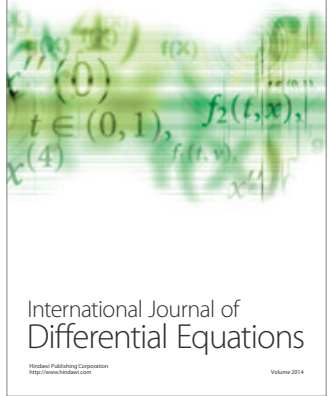
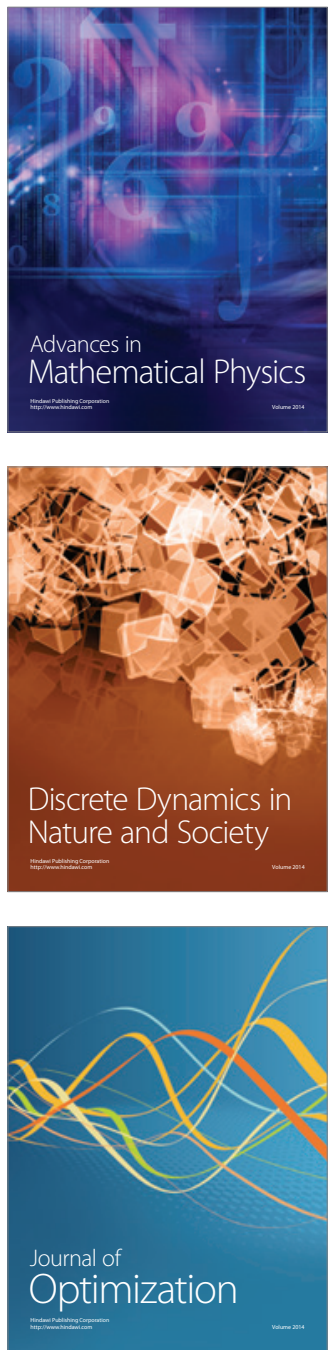